\title{
Research on Privacy Protection Approach for Cloud Computing En- vironments
}

\author{
Xiaohui $\mathrm{Li}^{1,2}$, Hongxing Liang ${ }^{3}$ and Dan Jia ${ }^{1}$ \\ ${ }^{1}$ College of Electrical and Information Engineer, Liaoning University of Technology \\ Jinzhou, Liaoning, 121001, China \\ ${ }^{2}$ Computer Science and Technology, Beijing University of Technology, \\ Beijing 100124, China \\ ${ }^{3}$ Jinzhou 65631 Force, Jinzhou, Liaoning 121001, China \\ lixiaohui@emails.bjut.edu.cn,lhxlxh@163.com
}

\begin{abstract}
Cloud computing is a convenient and cost efficient way that refers to the running of software over a network, as on a local computer. But, what is cheap and easy, isn't always safe. Storing important data on cloud means that you give up a certain control of privacy. Privacy protection is a very important issue in cloud computing. In this paper,we focus on the need of cloud users managed services and propose an approach for data protection based trust mechanism, which using adaptive mapping resources based on trust to ensure the user data protection initiative, and data security. The analysis shows that the cloud user can independently controls the content and manner of services by using the approach, which can achieve the free deployment of resources and the balance of user data protection and service provided.
\end{abstract}

Keywords: Cloud computing, privacy, security, trust

\section{Introduction}

Cloud computing can be well positioned to meet the needs of the users anytime, anywhere access to resources and transaction processing, in this computing architecture, and its essence is to make the data more and more exposed to the hazardous environment. Storing important programs and files on the cloud means that you give up a certain level of control and your privacy. Platform can be updated, perhaps you would be forced to figure them out all over again, or worse, cooperation between the cloud platform would mean unlawful access by the former. The user in cloud computing requirements at any time, any place can be carried out safely data access, Cloud has brought us unprecedented service experience at the same time, as the shadow of the user data security issues.

The solution to get the balance of user privacy protection and services in cloud computing is the key to promote its application. Trust as an important mean of security technology, which takes on an integral role from the beginning to the transmission of the mobile user information interaction. Authorization, access control and other security mechanisms are often accompanied by the provision of user privacy information in the cloud computing environments, users do not know their own interactive objects utmost prominence of security concerns. How to build trust between the user and the service provider, and to reconcile the balance between user privacy and service access? Approachs based on trust in privacy protection service-oriented technology will characterize user privacy trust granularity, and according to its variability and dynamic establish mapping relation- 
ship; based access control approach according to the security policy set to control access to privacy information, dynamic adjustment and setting of user privacy information and access granularity. The above studies focus considering the security threats posed by the malicious acts by the service requesting party and do not consider the security threats posed by the service provider. Trust between entities in the cloud computing environment assumptions no longer exist, from the point of view of the protection of user privacy, the above approaches are not applicable.

In this article, we focus on user data protection issues in cloud computing, propose a trust-based approach to data protection, while dynamically generate trust relationship to release its own data to achieve balance of privacy protection and services provided.

The rest of this paper is organized as follows. In Section 2, we describe some related work. In Section 3, we explain our approach for privacy protection in two aspects: one is to relevant definitions and the other is algorithm description. We also give an application scenario for better illustration of our approach. Finally, we conclude this paper in Section 4.

\section{RELATED WORK}

In the commodity market model, resource owners specify their service price and charge users according to the amount of resource they consume. Services are priced in such a way that supply-and-demand equilibrium is maintained. Which has two actors: The resource broker (working for the user) is denoted by B, Which can carry out the following steps for executing applications:

1. the broker identifies service providers;

2. it identifies suitable resources and establishes their prices;

3. it selects resources that meet its utility function and objectives;

4. it uses resource services for job processing and issues payments as agreed;

In the model, resource consumer only can depend on the specifed price of resource provider as a parameter, bargaining is not permitted.

Nash Equilibrium Theory does not require the asumption of individual rationality and noncooperation with the three elements: participants collection $\mathrm{O}$, strategy space $\mathrm{R}$ and the utility function $\mathrm{T}$, its process in chronological order provides as follows:

1 In accordance with the probability distribution $o\left(t_{i}\right)$ for the sender S type, which was established for each i and select $t_{i}$ from the type of a viable space;

2 Client observed $t_{i}$, select a transmission $m_{j}$;

3 Server observe the $m_{j}$, and select an action $a_{k}$ from the feasible action set;

4 The revenue of the two sides, respectively, $x c v v$ and $q_{r}\left(s_{i}, l_{j}, y_{k}\right)$.

Wherein the requirements of the B for each of the $m_{j}$ given posterior inference with probability distribution $p\left(t_{i} / m_{j}\right)$, the recipient's $a_{k}$ to maximize revenue, empathy for the transmission signal $\mathrm{S}$, for a given policy, its value is the optimal.

\section{Data Protection Model based on Trust}

\subsection{Approach Description}

In the cloud computing environment, service providers and cloud user information closely related to their private information, the user according to the degree of trust in the service, and hope to get the safe use, therefore access to information competition between service providers. Assume that private information of the service provided by the user to provide the correspond- 
ing service, but the user can not directly determine the true quality of the service and to initially approximate inference can only be made in accordance with the commitment of the service provider. In other words, from the point of view of the protection of user security considerations, users enjoy information superiority recipient $\mathrm{R}$, and service providers in the asymmetric information disadvantage, as the sender $\mathrm{S}$. There is certain mapping between privacy information release and service obtained the release, which is to be addressed in this article.

Cloud users based on attributes of their own resources or statistical information on the history of service selection behavior, develop appropriate time factors and spatial factors to quantify privacy degree rules table. Cloud users based resource properties will be divided into n privacy periods, for each privacy interzone develop appropriate Service demand interval, the implication is that cloud users at time of service, if, then randomly generates the time factor to quantify the value of trust. For each time period, the time period when the history of a certain number of service providers, the statistics of the time period and the total number of times to provide a number of fraud, the probability of obtaining fraudulent period .

According to privacy degree to provide services, mapping relationship as shown in Table 1:

\section{Table 1. Privacy and Service Mapping Table}

\begin{tabular}{|l|r|r|r|r|c|}
\hline $\begin{array}{l}\text { Privacy } \\
\text { degree }\end{array}$ & {$\left[t_{0}, t_{1}\right)$} & {$\left[t_{1}, t_{2}\right)$} & {$\left[t_{2}, t_{3}\right)$} & $\cdots$ & {$\left[t_{n-1}, t_{n}\right]$} \\
\hline $\begin{array}{l}\text { Service } \\
\text { demand } \\
\text { interval }\end{array}$ & {$\left[T_{0}, T_{1}\right)$} & {$\left[T_{1}, T_{2}\right)$} & {$\left[T_{2}, T_{3}\right)$} & $\ldots$ & {$\left[T_{n-1}, T_{n}\right]$} \\
\hline $\begin{array}{l}\text { Service } \\
\text { fraud } \\
\text { probability }\end{array}$ & $p_{0}$ & $p_{1}$ & $p_{2}$ & $\ldots$ & $p_{n-1}$ \\
\hline
\end{tabular}

Collection of the program participants \{service provider, the user\}, service providers to provide services, quality of service is high $(\mathrm{G})$ and $(\mathrm{D})$ two types, and the selected signal is the trust $\mathrm{X}$ ranges from 0 to $1\{\mathrm{x}: 0 \leq \mathrm{x} \leq 1\}$, their specific trust value is based on the direct trust $\left(\mathrm{X}_{\mathrm{z}}\right)$ and feedback trust $\left(\mathrm{X}_{\mathrm{f}}\right), \mathrm{X}=\mathrm{W}_{1} \times \mathrm{X}_{\mathrm{z}}+\mathrm{W}_{2} \mathrm{X}_{\mathrm{f}}$, where $\mathrm{W}_{1}$ andW $\mathrm{W}_{2}$ said $\mathrm{X}_{\mathrm{z}}$ and $\mathrm{X}_{\mathrm{f}}$ of the right weight; users decide their own privacy information the release of granularity $\mathrm{Y}-\mathrm{C}(\delta, \mathrm{x})$ said services provided type, $\mathrm{C}(\delta, \mathrm{x})$ table type for $\delta$ the services provided by the trust for the cost of the case, its value depends on the correspondence relationship of confidence and the service type; $\mathrm{H}$ $(\delta, \mathrm{x})$ indicates the type of service provided by the user are provided service gains; service provider's revenue as $\mathrm{Y}-\mathrm{C}(\delta, \mathrm{x})$, the gains of the service users for $\mathrm{H}(\delta, \mathrm{x})-\mathrm{Y}$.

Signal theory-based research in cloud computing adverse selection between service users and providers, with incomplete information based on the expected return of the mobile user privacy (in favor of the security of the service provider) service providers to take the initiative to abandon malicious deception motivation; mobile user can get the quality of service expectations and trust-based release privacy information, enabling mobile user's privacy protection.

\subsection{Program Structure}

Interactive service providers and mobile users' privacy information release decisions, according to the a priori probability distribution for the signal sender $S$ type can be selected from the line-type space, which type of collection $T=\left\{t_{d}, t_{g}\right\}$ indicates that the service quality low and high; then $\mathrm{S}$ from the signal set $\mathrm{M}=\left\{\mathrm{m}_{\mathrm{d}}, . ., \mathrm{m}_{\mathrm{g}}\right\}$ select a transmit signal, which signal set $\mathrm{m}_{\mathrm{d}}, \mathrm{m}_{\mathrm{g}}$ respectively, apply for a low level of privacy information and the application of a high level of 
privacy information; mobile user observations feasible action set privacy information and refused to provide privacy information; choose a behavior and calculate the two sides revenue.

In this scheme, the cloud user based on the services provided by the trust to determine the release of privacy information granularity, from the introduction of signal theory, the service providers to maximize their own interests, needs to take the initiative to provide honest services model service revenue management, service delivery, trust evaluation, trust, decision-making, privacy and information provided module, specific structure shown in Figure 1.

Implementation processes of the program are as follows:

(1) the user $U$ according to the probability distribution signal selection and make the appropriate services according to their own abilities t;

(2) the service provider $\mathrm{S}$ observed $\mathrm{t}$ from the corresponding set $P_{\text {upd }}=\left(\Omega_{i}, G\left(l_{i}\right)\right.$, sig $\left.{ }_{s k}(G(R)), R^{\prime}\right)$, select send a m and given initial trust;

(3) the recipient $\mathrm{R}$ observed $\mathrm{m}$ corresponding service provides assessment, including trust evaluation, trust, decision-making and feasible action set update $=\left(M, i, l_{i}^{\prime}, \sigma_{i}^{\prime}\right)^{\prime \prime}$ to select the appropriate action trust, sends it to the CSP;

(4) Enforcement action space A service revenue for rational decision-making cloud users, until both maximize the return on the end of the game process. The probability of assuming cloud services to provide the level of quality receives the request; replaces the block ${ }^{n_{i}}$ with ${ }^{n_{i}^{\prime}}$ and outputs $F^{\prime}$; replaces $\sigma_{i}$ with ${ }^{\sigma_{i}^{\prime}}$;

(5) responds the user with an evidence for this operation, where ${ }^{\Omega_{i}}$ is for the verification of

$n_{i}$.the $d_{p}$, said users of the server to provide a high level of privacy, $g_{p}$ expressed low levels of privacy information; $i_{g}$ provide high-quality service users gain $i_{d}$ said to provide low-quality service user gains; $\mathrm{p}\left(\mathrm{g} / \mathrm{g}_{\mathrm{p}}\right), \mathrm{p}\left(\mathrm{d} / \mathrm{g}_{\mathrm{p}}\right)$ denote the conditional probability of services for high privacy level of quality of service, users select the release of the privacy of revenue.

\subsection{Algorithm Description}

Depends on the service to apply for a balance between the cloud user privacy protection and access to privacy, the level of quality of service, the relationship between both sides revenue. Bayesian equilibrium point is that the parties to simply observe the behavior of others to modify their subjective probability of the latter characteristics, and thus choose their own actions, thus proving that cloud service providers corresponding combination and decision-making constitutes a perfect Bayesian equilibrium, the analysis process is as follows:

First analysis of the mobile user's decision-making, the initial trust service providers take the initiative to provide mobile users signal private information to provide the mobile user receives the signal selected action, specific actions, including service quality evaluation and correction priori probability. If the service provider application to the high-level privacy information, the mobile user to select the expected revenue issued $\mathrm{E}_{\mathrm{p}}=\mathrm{p}(\mathrm{H} / \mathrm{Y}) \mathrm{I}(\mathrm{H}, \mathrm{Y})+(1-\mathrm{P}(\mathrm{H} / \mathrm{Y})) \mathrm{Z}(\mathrm{E}, \mathrm{Y})$, $\mathrm{E}_{\mathrm{p}}=\left(\mathrm{i}_{\mathrm{g}}-\mathrm{p}_{\mathrm{g}}\right)>0$; Similarly, if the service to apply for low privacy quantity, the cloud user to select payment expected revenue for $E_{p}=\left(i_{d}-p_{d}\right)>0$;. 


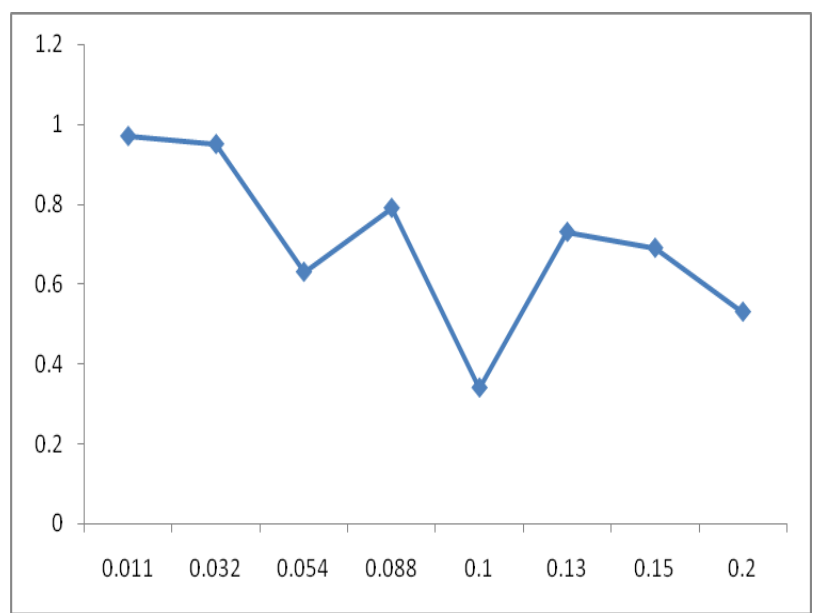

Figure 1. The Amount of Information Released with Optimal Trust

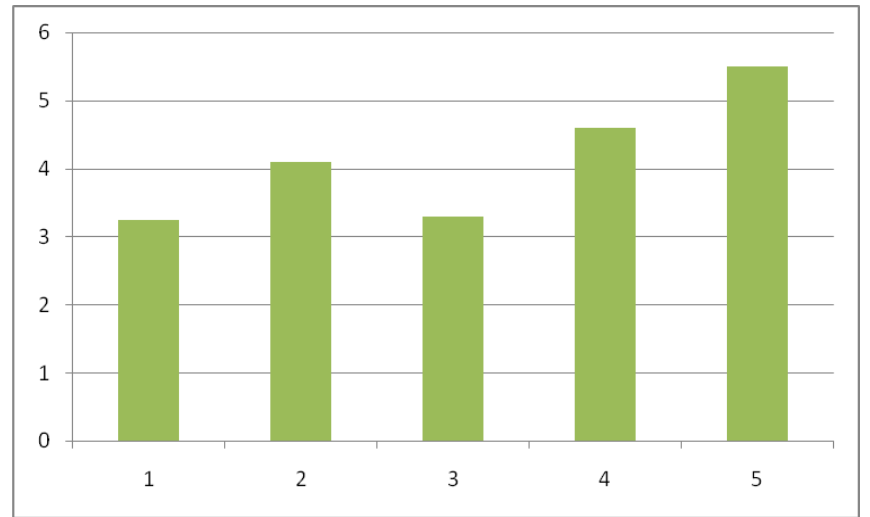

Figure 2. Service Permission Change

And analysis of the decisions of the service provider, the service provider revenue maximization conditions for max $[\mathrm{H}(\delta, \mathrm{x})-\mathrm{C}(\delta, \mathrm{x})]$, the high quality of service to apply the proceeds of the high level of privacy $\mathrm{p}_{\mathrm{g}} \geq \mathrm{p}_{\mathrm{d}}$; low service quality to provide the requested high-level privacy gains, $\mathrm{Z}(\mathrm{F})-\mathrm{D}(\mathrm{F} \times(\mathrm{E}))>\mathrm{Z}(\mathrm{H})-\mathrm{D}(\mathrm{F}-, \mathrm{x}(\mathrm{H}))$, that this decision is not suitable for only the application of the low-level privacy information in order to achieve optimal.

Suppose two participants in the incomplete information case the optimal trust shown in Figure 1, where the horizontal axis represents the degree of the sender R's trust, the vertical axis represents the release of the privacy of the recipient $S$ particle size, can be seen participating under balanced Strategic correction priori probability, and according to a given probability distribution on the set of information and others involved in the follow-up strategy to maintain optimal action, follow the above strategy Bayes rule to meet the needs of path information on the balanced and rational sequence satisfy the refined Tony Yates balancing requirements. Weigh trust and security, service providers and get the two sides will achieve perfect Bayesian equilibrium, that the services of a high quality of service will require high privacy, low quality level of service will consciously apply for a low level of privacy, and mobile privacy, users must release service application level of service to apply the amount of privacy can completely reflect the true quality of service.

\section{Conclusion}


In this paper, we proposed a user data protection approach in cloud computing environment in which we define trust metrics as a dynamic and flexible process that offers a new perspective for decision-making. The analysis shows that the performance of our approach is obvious and it can help achieve less privacy loss and more benefits. That the high fraud costs make the services and privacy provided both sides to get the maximum benefit, this approach can effectively protect users' privacy and containment malicious service side, from another point of view, it is also beneficial to the security of the service side. The approach proposed in this paper exposes us to a number of possible research directions in the future. One direction that we will implement the approach to study the performance issues. We will take is to refine our approach to support multi-services and to help us further optimize the approach.

\section{Acknowledgments}

The work in this paper has been supported by National Natural Science Foundation of China (Grant No.61272500).

Beijing Natural Science Foundation (4142008).

Pre-launch of Beijing City Government Major Tasks and District Government Emergency Projects (Z131100005613030)

\section{References}

[1] M. A. Moharrum and M. Eltoweissy, "A Study of Static Versus Dynamic Keying Schemes in Sensor Networks", Proc. of the 2nd ACM Int'l Workshop on Performance Evaluation of Wireless Ad Hoc, Sensor, and Ubiquitous Networks. New York, USA: ACM Press, (2005), pp. 122-129.

[2] L. Sweeney, "Achieving k-Anonymity Privacy Protection Using Generalizatio n and Suppressio n", International Journal of Uncer tainty, Fuzziness and Knowledge Based Systems, vol. 10, no. 5, (2002), pp. 571- 588.

[3] L. Ibraimi, M. Petkovic, S. Nikova, P. Hartel and W. Jonker, "Ciphertext-Policy attribute-based threshold decryption with flexible delegation and revocation of user attributes", Technical Report, Centre for Telematics and Information Technology, University of Twente, (2009).

[4] D. Nurmi, R. Wolski and C. Grzegorczyk, "Eucalyptus: A Technical Report on an Elastic Utility Computing Archietcture Linking Your Programs to Useful Systems Tech.”, Rep. 2008-10, University of California, Santa Barbara, (2008) October.

[5] C. Castelfranchi, R. Falcone and G. Pezzulo, "Integrating trustfulness and decision using fuzzy cognitive maps", Trust Management, LNCS, vol. 2692, (2003), pp. 195-210.

[6] D. D. Salber and G. Abowd, "A Conceptual Framework and a Toolkit for Supporting the Rapid Prototyping of Context-Aware Applications", Special issue on Context-Aware Computing in the Human-Computer Interaction (HCI) Journal, vol. 16, no. 2-4, (2001), pp. 97-166.

[7] B. J. Jansen, A. Spink and T. Saracevic, "Real Life, Real Users, and Real Needs: A Study and Analysis of User Queries on the Web", Information Processing and Management, vol. 36, no. 2, (2000), pp. 207-227.

[8] A. N. Ramjattan and P. A. Gross, "A Kalman filter model for an integrated land vehicle navigation system", The Journal of Navigation, vol. 48, no. 2, (1995), pp. 293-302.

[9] R. Gibbons, “An Introduction To Applicable Game Theory”, Journal of Economic Perspectives, vol. 11, no. 1, (1997), pp. 127-141.

[10] M. Blaze, J. Feigenbaum and J. Lacy, "Decentralized Trust Management", Proceedings of the 17th Symposium on Security and Privacy, (1996), pp. 164-173.

[11] P. B. Ardagnad, "Adaptive service composition in flexible processes", IEEE Transactions on Software Engineering, vol. 33, no. 6, (2007), pp. 369-384.

[12] M. Spence, "Job Market Signaling”, The Quarterly Journal of Economics, vol. 87, no. 3, (1973), pp. 355-374.

\section{Author}




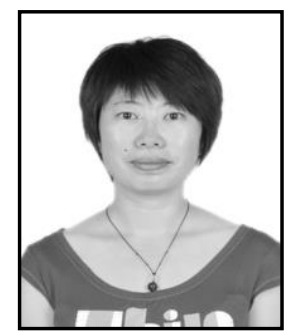

Xiaohui Li is currently a lecturer of the college of Electrical and Information Engineer at Liaoning University of Technology and a Ph.D. candidate in the College of Computer Science and Technology at Beijing University of Technology. Her research interests include network security and trust management. Email: lixiaohui@emails.bjut.edu.cn 
International Journal of Security and Its Applications Vol. 9, No. 3 (2015) 